\title{
Magnetic hysteresis scaling behavior in terbium and holmium
}

\author{
Satoru Kobayashi* \\ Department of Materials Science and Engineering, Faculty of Engineering, Iwate University, Ueda 4-3-5, Morioka 020-8551, Japan
}

(Received 8 November 2011; published 20 December 2011)

\begin{abstract}
Hysteresis scaling for magnetic minor loops has been examined in the heavy rare-earth metals $\mathrm{Tb}$ and Ho, varying the temperature and magnetic-field amplitudes. In addition to the low-temperature ferromagnetic phase below $T_{\mathrm{c}}$, the scaling law between the hysteresis loss and remanent flux density with an exponent of $\sim 1.3$ was observed in the higher-temperature helical antiferromagnetic phase for both metals. The coefficient which reflects the pinning fields of the domain walls decreases with increasing temperature below $T_{\mathrm{c}}$, but it exhibits a sharp increase after the onset of the helical antiferromagnetic phase. The observations above $T_{\mathrm{c}}$ were explained as due to the irreversible motion of spiral domain walls, unlike the $180^{\circ}$ Bloch walls that are typical in the ferromagnetic phase.
\end{abstract}

DOI: 10.1103/PhysRevB.84.224418

PACS number(s): 75.60.Ej, 75.60.Ch

\section{INTRODUCTION}

Magnetic hysteresis scaling associated with irreversible domain wall motion has been widely and extensively studied because of its technological application and fundamental physical importance. ${ }^{1,2}$ In the case of bulk ferromagnets, the empirical Steinmetz law, which is a power-law relation between hysteresis loss and maximum flux density, has continued to contribute in the engineering field to magnetic quality examinations, the design of motors, etc. ${ }^{3,4}$ However, the law is generally valid under the dominant contribution of irreversible Bloch wall motion and its applicability is therefore limited in the intermediate magnetization range for soft ferromagnetic materials. Recent detailed reinvestigations on hysteresis scaling revealed another hysteresis scaling law and also its widely usable magnetic characterization for semihard ferromagnetic materials ${ }^{5}$ and small ferromagnetic particles embedded in a nonmagnetic matrix. ${ }^{6,7}$ The hysteresis loss was found to be also power-law related to the remanent flux density with an exponent of 1.3-1.4; this scaling law can only reflect the irreversible mechanism of domain walls even under a significant contribution of the reversible process due to magnetization rotation and domain wall displacement.

Quite recently we investigated the hysteresis scaling of minor $B-H$ loops for a rare-earth metal dysprosium. In addition to the ferromagnetic (FM) phase, a scaling power law between the hysteresis loss and remanent flux density was found to hold true even in the higher-temperature helical antiferromagnetic (HAM) phase with no net spontaneous magnetization. ${ }^{8}$ The power-law exponent of the HAM phase was $\sim 1.25$, which is very close to values of the FM phase and conventional soft ferromagnetic materials. ${ }^{5}$ These observations indicate a universal hysteresis scaling due to an irreversible process of spiral domain walls, which separate HAM domains with oppositely rotating spins. ${ }^{9-12}$ The scaling coefficient gave information about the pinning fields for spiral domain walls, which may be related with the turn angle of the HAM structure. These findings open up the possibility of investigating the pinning mechanism using hysteresis scaling, not only for Bloch walls that are typical in ferromagnets, but also for other types of domain walls present in antiferromagnetic structures such as HAM, conical, and sinusoidal ones.
In this paper, we have examined the scaling behaviors of magnetic hysteresis loops for the heavy rare-earth metals $\mathrm{Tb}$ and Ho. Both metals exhibit FM and incommensurate HAM phases at low and high temperatures, respectively, but their associated magnetic structures differ from each other. ${ }^{13} \mathrm{~Tb}$ exhibits a HAM structure with spins confined on the hexagonal basal plane below $T_{\mathrm{N}} \sim 230 \mathrm{~K}$. As the temperature decreases, a turn angle between the adjacent planes $\theta_{0}$ slightly decreases from $20.5^{\circ}$ to $17^{\circ}$, and then rises to $18.5^{\circ}$, and finally a magnetic phase transition toward the FM phase takes place at $T_{\mathrm{c}} \sim 220 \mathrm{~K}$. Ho also exhibits a HAM structure below $T_{\mathrm{N}} \sim 130 \mathrm{~K}$, where $\theta_{0}$ decreases from $\sim 50^{\circ}$ at $T_{\mathrm{N}}$ to $30^{\circ}$ with decreasing temperature. Below $T_{\mathrm{c}} \sim 20 \mathrm{~K}$, Ho exhibits a conical antiferromagnetic structure with a half-cone angle of $\sim 80^{\circ}$, which is associated with a small ferromagnetic component along the $c$ axis. For both metals, the hysteresis scaling properties of $B-H$ loops in the FM and HAM phases have been examined and discussed in detail.

\section{EXPERIMENT}

Toroidal samples of polycrystalline $\mathrm{Tb}$ and Ho were cut from an ingot with a purity of $99.9 \%$ by an electric discharge machine and were then polished to eliminate oxidation layers; the samples had external and internal diameters of 9 and $7 \mathrm{~mm}$, respectively, and a thickness of $5 \mathrm{~mm}$. Flux-density $(B)$-field $(H)$ loops were measured with a conventional fluxmetric method. Here 125-turn exciting and 100-turn detecting coils of copper wires were wound around the sample to generate a cyclic magnetic field in the circumferential direction and pick up the induced voltage due to magnetization, respectively. The sampling rate was $2000 \mathrm{~Hz}$. $B$ - $H$ loops with various field amplitudes $H_{\mathrm{a}}$ were measured by a step-by-step increase of $H_{\mathrm{a}}$, keeping the speed of the applied field $d H / d t$. The maximum value of $H_{\mathrm{a}}$ was $\sim 8 \mathrm{kA} / \mathrm{m}$. Before the measurements, the sample was demagnetized with a decaying alternating magnetic field of $4 \mathrm{~Hz}$. Various values of $d H / d t$ in the range of $8-128 \mathrm{kA} / \mathrm{m} / \mathrm{s}$ were tested. Since the results were weakly dependent on $d H / d t$, the eddy current effect was not important. The results for $d H / d t=64 \mathrm{kA} / \mathrm{m} / \mathrm{s}$ for Ho and $16 \mathrm{kA} / \mathrm{m} / \mathrm{s}$ for $\mathrm{Tb}$ will be given in this study. The sample was placed in a He-gas closed-cycle refrigerator and cooled 

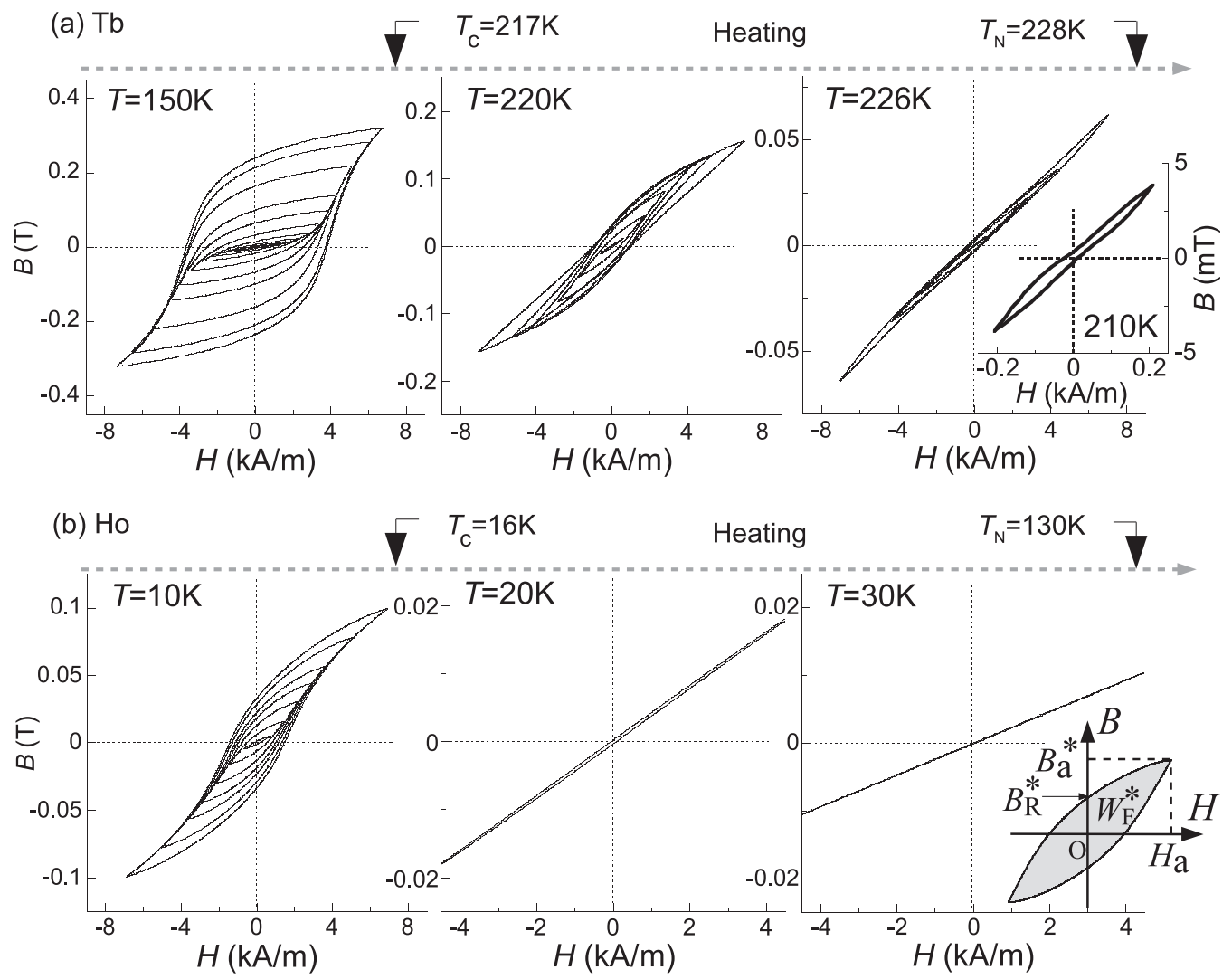

FIG. 1. $B$ - $H$ loops with various field amplitudes $H_{\mathrm{a}}$, taken at different temperatures on heating for (a) Tb and (b) Ho. The inset in (a) shows a low-field $B-H$ loop at $T=210 \mathrm{~K}$ for Tb, taken on heating. The inset in (b) shows the parameters of a $B-H$ loop; $B_{\mathrm{a}}^{*}, B_{\mathrm{R}}^{*}$, and $W_{\mathrm{F}}^{*}$ are the maximum flux density, remanent flux density, and hysteresis loss, respectively.

down to $5 \mathrm{~K}$. Measurements were performed at intervals of 1 or $2 \mathrm{~K}$ on heating from $5 \mathrm{~K}$ or cooling from a temperature in the paramagnetic $(\mathrm{PM})$ phase above $T_{\mathrm{N}}$. The temperature was stabilized at each measuring temperature to within $0.05 \mathrm{~K}$.

\section{EXPERIMENTAL RESULTS}

\section{A. Behavior of $B-H$ loops}

Figures 1(a) and 1(b) show a set of $B-H$ loops with various field amplitude $H_{\mathrm{a}}$, taken at various temperatures on heating for $\mathrm{Tb}$ and $\mathrm{Ho}$, respectively. For each loop with different $H_{\mathrm{a}}$, the parameters $B_{\mathrm{a}}^{*}, B_{\mathrm{R}}^{*}$, and $W_{\mathrm{F}}^{*}$, defined as those in the inset in Fig. 1(b), were obtained. In Figs. 2(a) and 2(c), the temperature dependence of minor-loop initial permeability $\mu_{\mathrm{m}}$ for $\mathrm{Tb}$ and Ho at temperatures around $T_{\mathrm{c}}$, taken on heating and cooling, is given. Here, $\mu_{\mathrm{m}}$ was obtained from a linear part of the $B_{\mathrm{a}}^{*}-H_{\mathrm{a}}$ curves for $B_{\mathrm{a}}^{*}$ below $2 \times 10^{-3}$ T. From local maxima of $\mu_{\mathrm{m}}^{*}, T_{\mathrm{N}}$ and $T_{\mathrm{c}}$ were determined for each sample: $T_{\mathrm{N}}=$ $228 \mathrm{~K}$, and $T_{\mathrm{c}}=217$ and $216 \mathrm{~K}$ for heating and cooling, respectively, for $\mathrm{Tb} ; T_{\mathrm{N}}=130 \mathrm{~K}$ [not shown in Fig. 2(c)] and $T_{\mathrm{c}}=16 \mathrm{~K}$ for both heating and cooling, for Ho. A thermal hysteresis for $\mu_{\mathrm{m}}$ was less than $1 \mathrm{~K}$ at around $T_{\mathrm{c}}$ for both samples, whereas no thermal hysteresis was detected at around $T_{\mathrm{N}}$.

For $\mathrm{Tb}$, a hysteresis behavior typical for ferromagnetic materials was observed below $T_{\mathrm{c}}$ [Fig. 1(a): $T=150 \mathrm{~K}$ ].
The value of $B_{\mathrm{a}}^{*}$ is considerably smaller than the saturation value $[\sim 3.39 \mathrm{~T}$ at $T=0 \mathrm{~K}$ (Ref. 13)], primarily due to large basal anisotropy and the polycrystalline nature of the sample. With increasing temperature, $B_{\mathrm{a}}^{*}$ gradually decreases, accompanying the narrowing of the loop width. Even after the onset of the HAM phase at $T_{\mathrm{c}}$, however, a hysteresis behavior, associated with a finite hysteresis loss, was clearly observed and persists up to a temperature near $T_{\mathrm{N}}$ [Fig. 1(a): $T=220$ and $226 \mathrm{~K}]$. A quite similar temperature dependence of the hysteresis loops was also observed on cooling; the hysteresis loss starts to appear just below $T_{\mathrm{N}}$ on cooling. This less thermal hysteresis is reflected in the temperature dependence of $\mu_{\mathrm{m}}$ shown in Fig. 2(a). This result is in contrast to that for Dy, where a hysteresis behavior in the HAM phase was only observed on heating.

In the case of Ho, a similar temperature dependence of the $B-H$ loops to that for $\mathrm{Tb}$ was observed below $T_{\mathrm{c}}$ on heating. Above $T_{\mathrm{c}}$ the hysteresis loss was drastically reduced, but the hysteresis behavior seems to remain even in the HAM phase [Fig. 1(b): $T=20 \mathrm{~K}$ ]. Within our experimental accuracy, the hysteresis loss was not detected at approximately a temperature of $21 \mathrm{~K}$. On cooling from a temperature above $T_{\mathrm{N}}$, the hysteresis loss appears at a temperature that is very close to $T_{\mathrm{c}}$ and rapidly increases below $T_{\mathrm{c}}$. The observed hysteresis loss in the HAM phase only on heating is reflected in a higher value of $\mu_{\mathrm{m}}$ just above $T_{\mathrm{c}}$, as shown in Fig. 2(c). 

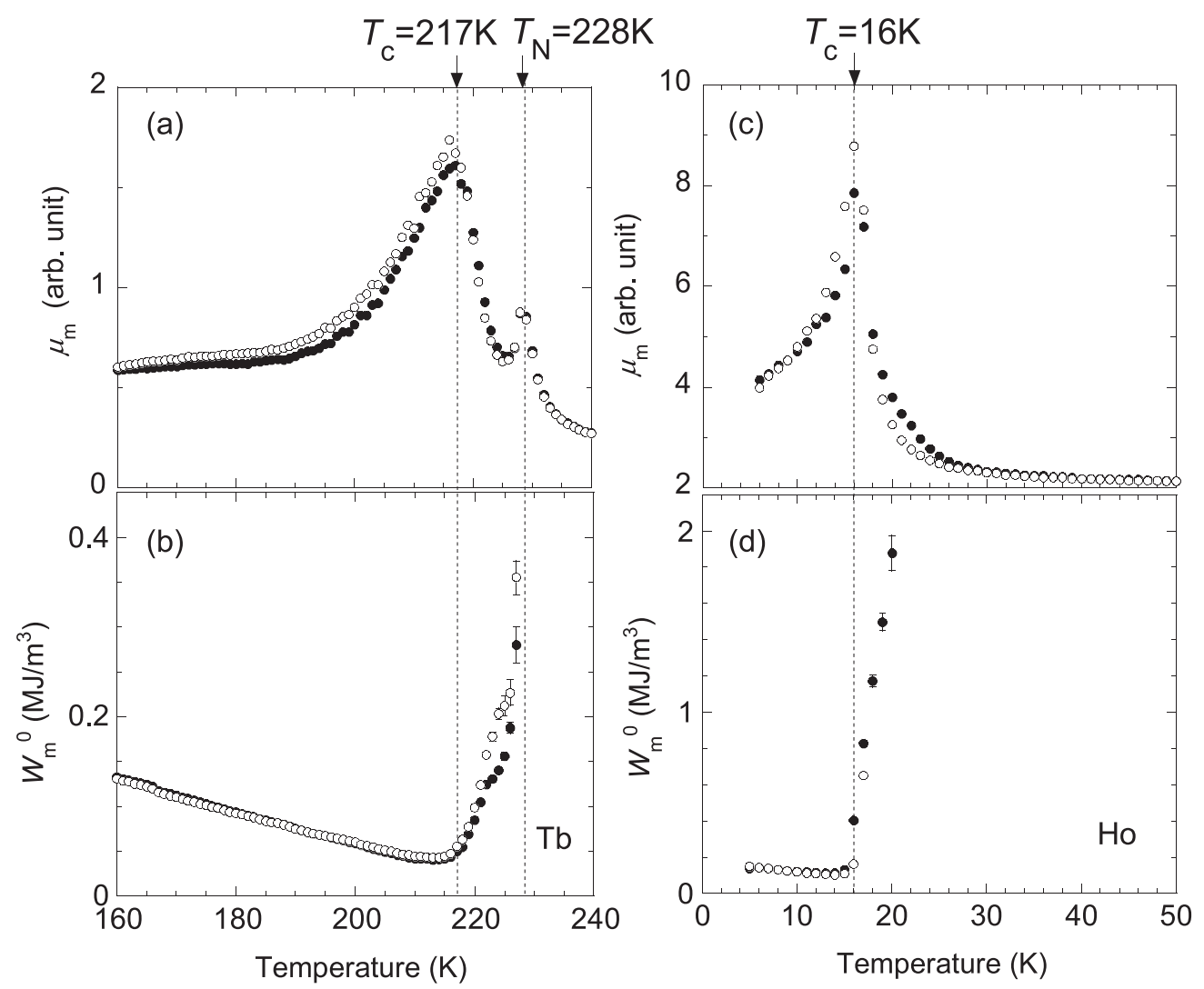

FIG. 2. Minor-loop initial permeability $\mu_{\mathrm{m}}$ and coefficient of a scaling power law $W_{\mathrm{m}}^{0}$ as a function of temperature for Tb [(a), (b)] and Ho [(c), (d)] near $T_{\mathrm{c}}$. The solid and open circles represent the data taken on heating and cooling, respectively.

\section{B. Hysteresis scaling analysis}

In this paper, we focused on a scaling power law of the $B-H$ loops between $W_{\mathrm{F}}^{*}$ and $B_{\mathrm{R}}^{*}$. Unlike the conventional Steinmetz law for soft ferromagnets, which is a power law between $W_{\mathrm{F}}^{*}$ and $B_{\mathrm{a}}^{*}$, the law between $W_{\mathrm{F}}^{*}$ and $B_{\mathrm{R}}^{*}$ can extract a scaling behavior for irreversible wall motion even under the significant influence of a reversible mechanism due to domain wall motion and magnetization rotation. ${ }^{5-7}$

Figures 3(a) and 3(b) show double logarithmic plots of the $W_{\mathrm{F}}^{*}-B_{\mathrm{R}}^{*}$ curves taken at various temperatures on heating for $\mathrm{Tb}$ and Ho, respectively. For both $\mathrm{Tb}$ and Ho, the curves exhibit straight lines below $T_{\mathrm{c}}$ in a low and medium $B_{\mathrm{R}}^{*}$ range over two orders of magnitude $\left(10^{-4}-10^{-2} \mathrm{~T}\right)$. Even in the HAM phase above $T_{\mathrm{c}}$ the linearity persists in a low $B_{\mathrm{R}}^{*}$ range, keeping the slope almost constant. The linear behavior indicates a powerlaw scaling behavior of the $W_{\mathrm{F}}^{*}-B_{\mathrm{R}}^{*}$ curves.

In order to extract hysteresis scaling properties due to an irreversible mechanism, the observed curves were leastsquares fitted to a power law, given by

$$
W_{\mathrm{F}}^{*}=W_{\mathrm{m}}^{0}\left(B_{\mathrm{R}}^{*} / B_{\mathrm{s}}\right)^{n_{\mathrm{m}}},
$$

where $n_{\mathrm{m}}$ is a power-law exponent and $W_{\mathrm{m}}^{0}$ is a coefficient that is sensitive to the pinning field of the domain walls, and $B_{\mathrm{s}}$ is the normalization constant and was assumed to be the saturation magnetization of each metal: $B_{\mathrm{s}}=3.39$ and 3.87 $\mathrm{T}$ for $\mathrm{Tb}$ and Ho, respectively. ${ }^{13}$ The fits yielded a quite similar value of $n_{\mathrm{m}}$ below and above $T_{\mathrm{c}}$ for both Tb and Ho: $1.32 \pm 0.04\left(T<T_{\mathrm{c}}\right)$ and $1.36 \pm 0.06\left(T>T_{\mathrm{c}}\right)$ for $\mathrm{Tb}$ and
$1.33 \pm 0.04\left(T<T_{\mathrm{c}}\right)$ and $1.32 \pm 0.05\left(T>T_{\mathrm{c}}\right)$ for Ho. Averaging these values for each metal gave $n_{\mathrm{m}}=1.32 \pm 0.05$ and $1.33 \pm 0.04$ for $\mathrm{Tb}$ and $\mathrm{Ho}$, respectively. The values for each metal are almost the same and close to 1.25 for Dy (Ref. 8) and 1.3-1.4 for soft ferromagnetic materials. ${ }^{5}$ This strongly indicates the presence of a universal hysteresis scaling behavior in the relation between the hysteresis loss and the remanent flux density.

In Figs. 2(b) and 2(d), the coefficient $W_{\mathrm{m}}^{0}$ as a function of temperature is shown for $\mathrm{Tb}$ and Ho, respectively. For both metals, $W_{\mathrm{m}}^{0}$ monotonically decreases with increasing temperature below $T_{\mathrm{c}}$, exhibits a local minimum just below $T_{\mathrm{c}}$, and then sharply increases in the HAM phase. This implies a drastic change in the pinning fields for the domain walls at $T_{\mathrm{c}}$. A temperature hysteresis, associated with a value of $W_{\mathrm{m}}^{0}$ on cooling greater than that on heating, was observed in the HAM phase for Tb.

\section{DISCUSSION}

For $\mathrm{Tb}$, full magnetic moments of $9.3 \mu_{\mathrm{B}}$ per atom are confined along the $a$ axis in the FM phase. ${ }^{13}$ In this case $180^{\circ}$ Bloch walls in which the magnetic moments rotate on the hexagonal basal plane separate antiphase FM domains, as in the case of the FM phase for Dy (Ref. 14) that is shown in Fig. 4(a). On the other hand, in the case of Ho, a small $1.7 \mu_{\mathrm{B}}$ ferromagnetic component along the $c$ axis of $10.3 \mu_{\mathrm{B}}$ full magnetic moments contributes to ferromagnetism below $T_{\mathrm{c}}$; the basal plane components are bunched in pairs close to 

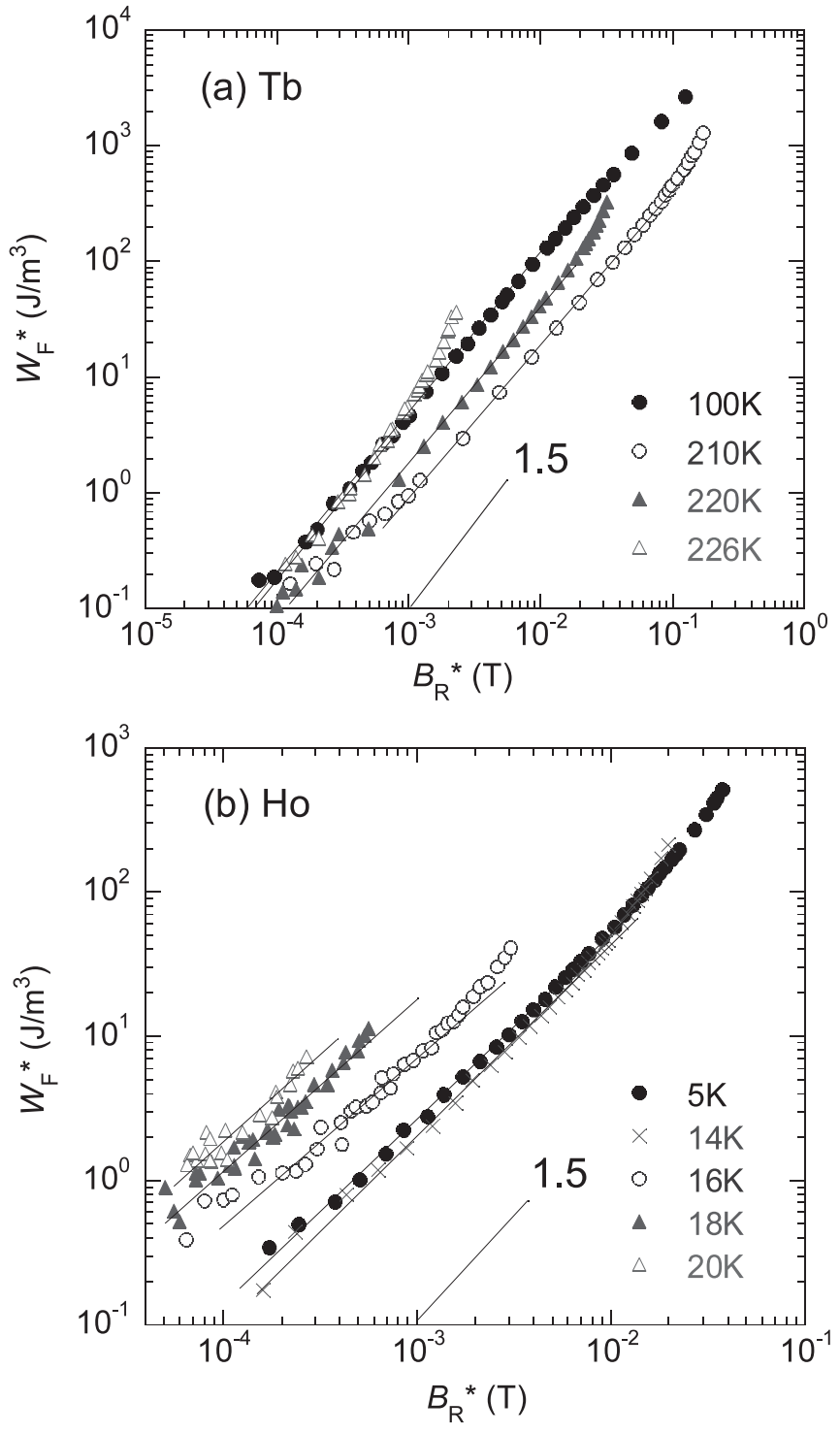

FIG. 3. Relationship between $W_{\mathrm{F}}^{*}$ and $B_{\mathrm{R}}^{*}$ at different temperatures on heating, plotted on a double logarithmic scale for (a) Tb and (b) Ho. The solid lines through the data denote least-squares fits. A slope of 1.5 is given for comparison.

an easy $b$ axis and a distorted helical ordering characterized by the wave vector $(1 / 6) c^{*}$ is formed. For such a conical structure, a $180^{\circ}$ Bloch wall, in which magnetic moments continuously rotate from one direction on an easy conical surface to some other direction on the different conical one, will also be a stable wall, as in the case of Gd (Refs. 15 and 16) that is shown in Fig. 4(b). According to our analysis which is based on Rayleigh law, ${ }^{5}$ a hysteresis scaling behavior of the $W_{\mathrm{F}}^{*}-B_{\mathrm{R}}^{*}$ relation will appear in a low and medium $B_{\mathrm{R}}^{*}$ range if the irreversible mechanism of domain walls exists. Irreversible motion of such Bloch walls results in the hysteresis loss in $B-H$ loops, yielding hysteresis scaling with a scaling exponent of $\sim 1.3$ that is similar to that of soft ferromagnetic materials. ${ }^{5}$ At high values of $B_{\mathrm{R}}^{*}$, on the other hand, the deviation of the $W_{\mathrm{F}}^{*}-B_{\mathrm{R}}^{*}$ curves was generally observed for both $\mathrm{Tb}$ and Ho (Fig. 3). This deviation reflects the appearance of another energy dissipation mechanism, possibly due to the irreversible rotation of magnetic moments. (a)

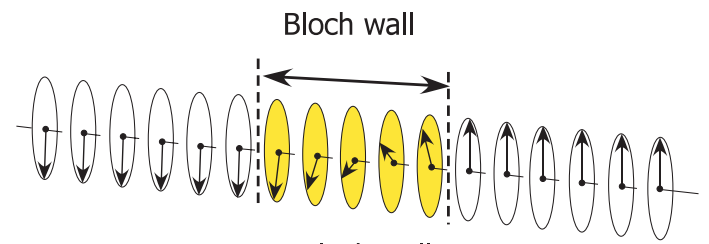

(b)

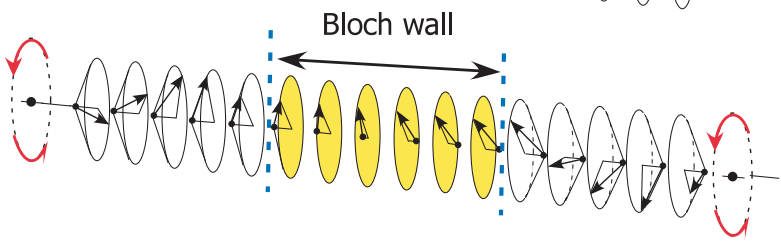

(c)

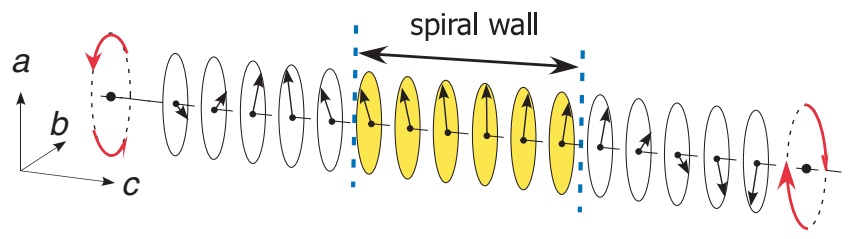

FIG. 4. (Color online) Schematic drawing of possible domain walls in the FM phase (a) for Tb, (b) for Ho, and (c) in the HAM phase. The arrows represent magnetic moments. In (b), the $c$-axis component of the magnetic moments changes its direction when passing through the Bloch wall, keeping the rotation direction on the basal plane. In (c), the spiral walls separate the HAM domains with magnetic moments rotating in an opposite direction.

Note that in the case of $\mathrm{Tb}$ a slight deviation from the hysteresis scaling behavior was also observed at low $B_{\mathrm{R}}^{*}$ below $10^{-3} \mathrm{~T}$ in a temperature range from $\sim 170 \mathrm{~K}$ to $T_{\mathrm{c}}$, as can be seen in the $W_{\mathrm{F}}^{*}-B_{\mathrm{R}}^{*}$ curve at $T=210 \mathrm{~K}$ in Fig. 3(a). Correspondingly, constriction of the $B-H$ loops such as that shown in the inset in Fig. 1(a) was detected. Previous work reported an anomalous increase of domain size ${ }^{17}$ as well as a reduction of low-field magnetic susceptibility ${ }^{18}$ in this specific temperature range; the former could be related to a domain nucleation, associated with a very weak basal plane anisotropy and nonzero spontaneous magnetization below $T_{\mathrm{c}}$, while the latter may be related to pinning and depinning processes for domain walls with mobile pinning centers such as impurity atoms. ${ }^{18}$ Stabilization of domain walls by such pinning centers frequently accompanies constriction of hysteresis loops, ${ }^{19}$ which is consistent with our observations. These mobile pinning centers may be much more reflected in the aftereffects of the $B-H$ loops in the high-frequency regime, and detailed measurements with high $d H / d t$ are planned.

On heating from below $T_{\mathrm{c}}$, the HAM structure with a propagation wave number along the $c$ axis is formed at $T_{\mathrm{c}}$. It will be formed through the nucleation of different ferromagnetic domains within preexisting ferromagnetic domains or through changes in the orientation of the magnetic moments within the Bloch walls. These processes lead to the formation of spiral domain walls separating the local HAM domains. ${ }^{11}$ Within the spiral wall, the turn angle of the magnetic moments may change continuously from a helical turn angle $+\theta_{0}$ to $-\theta_{0}$, as schematically shown in Fig. 4(c). These spiral domain walls are considered to behave as thin ferromagnetic layers and displace in a magnetic field so as to lower the Zeeman energy of the walls. This will give rise to net ferromagnetic moments along the direction of the applied field, in addition to those due to modifications of the local magnetic structure 
within the HAM domains. The irreversible motion of spiral domain walls under pinning fields will lead to a hysteresis loss in the HAM phase. As the temperature increases, a turn angle between adjacent layers increases for both metals. This change as well as an increase in thermal energy drastically reduce the number of spiral domain walls, diminishing the hysteresis loss at higher temperatures. At present, it is not clear why irreversible spiral wall motion can yield hysteresis scaling with a very similar exponent to that for Bloch walls. Nevertheless, this observation may suggest the presence of an underlying mechanism of domain wall pinning irrespective of the types of domain walls.

Here we note the temperature range in which spiral domain walls appear. In the case of Ho, spiral domain walls are formed only in the considerably narrow temperature range of $5 \mathrm{~K}$ above $T_{\mathrm{c}}$, compared with $\sim 50 \mathrm{~K}$ for Dy. This may be attributed to the helical nature of magnetic moments below $T_{\mathrm{c}}$ for Ho, because the HAM structure is easily formed at $T_{\mathrm{c}}$ without significantly changing the domain pattern. On the other hand, for $\mathrm{Tb}$ the observations imply the formation of a large number of spiral domain walls both on heating and cooling. A possible high concentration of small ferromagnetic inclusions ${ }^{20}$ may be eliminated because no hysteresis loss was observed above $T_{\mathrm{N}}$. Considering a high sensitivity of the HAM domain size to crystal purity, ${ }^{21}$ spiral domain walls can nucleate at defect sites also on cooling through $T_{\mathrm{N}}$. This may be a reason why the hysteresis loss was observed in the HAM phase on both heating and cooling. We also note that there exists no significant contribution of the distribution of domains with different $T_{\mathrm{c}}$ and $T_{\mathrm{N}}$, which may arise due to impurity atoms and/or a temperature gradient and results in the distribution of a pinning field over the sample; this is because $W_{\mathrm{m}}^{0}$, which is also sensitive to the distribution of the pinning field, ${ }^{7}$ exhibits no significant thermal hysteresis at around $T_{\mathrm{c}}$.

Now we discuss the behavior of $W_{\mathrm{m}}^{0}$, which is a sensitive indicator of a pinning field. ${ }^{5}$ For both metals, $W_{\mathrm{m}}^{0}$ decreases in the FM phase and shows a steep increase in the HAM phase with increasing temperature, as shown in Figs. 2(b) and 2(d). This behavior is very similar to that for Dy and may be interpreted as due to the same origin as follows. ${ }^{8}$ In the FM phase, the width of the Bloch walls is expected to be a few tens of atomic layers and is much smaller than a few hundred atomic layers for soft ferromagnetic materials because of a large sixfold basal anisotropy: $\sim 20$ atomic layers at $T=0 \mathrm{~K}$ for $\mathrm{Tb}$, with seven atomic layers for $\mathrm{Dy} .^{22}$ In this case, the energy of the Bloch walls depends on the center position with respect to the atomic lattice and the Peierls potential due to a periodic lattice that would contribute to domain wall pinning, in addition to a pinning mechanism that is due to lattice defects. ${ }^{22}$ With increasing temperature, this Peierls energy greatly decreases because of a decrease of the sixfold basal anisotropy ${ }^{23}$ and an increase of the thermal fluctuation, resulting in a decrease in $W_{\mathrm{m}}^{0}$ at higher temperatures below $T_{\mathrm{c}}$

In the HAM phase above $T_{\mathrm{c}}$, the anisotropy is small and the structure of the spiral wall would be mainly determined by interlayer exchange interactions. According to simple calculations, ${ }^{12,24,25}$ the width of the spiral wall is only a few atomic layers and decreases with a helical turn angle $\theta_{0}$ : for instance, approximately five atomic layers for Ho when $\theta_{0}=36^{\circ}$. Therefore, spiral walls will feel a periodical energy barrier against the wall motion, as does the Peierls potential in the FM phase, whose height increases with decreasing wall thickness. Since for both $\mathrm{Tb}$ and Ho $\theta_{0}$ increases with increasing temperature, ${ }^{13}$ the domain wall pinning will be strengthened at higher temperatures. This pinning mechanism, which depends on the helical turn angle, is consistent with an increase of $W_{\mathrm{m}}^{0}$ in the HAM phase for both metals. For $\mathrm{Tb}$, a significant temperature hysteresis of $W_{\mathrm{m}}^{0}$ was observed [Fig. 2(b)]; a thermal hysteresis of a turn angle and/or domain structure ${ }^{21}$ may be related to this observation. Future extensive theoretical work on the pinning mechanism for spiral domain walls will give a deep understanding of the behavior of $W_{\mathrm{m}}^{0}$ for rare-earth metals.

\section{CONCLUSION}

We have investigated a hysteresis scaling law between the hysteresis loss and remanent flux density in the heavy rareearth metals $\mathrm{Tb}$ and Ho. The scaling law was found to hold true in both FM and HAM phases with exponents of $1.32 \pm 0.05$ and $1.33 \pm 0.04$ for $\mathrm{Tb}$ and Ho, respectively. The values are close to 1.25 for Dy and 1.3-1.4 for soft ferromagnets, found previously, implying that the universal pinning mechanism does not depend on the types of domain walls, i.e., Bloch and spiral walls. The coefficient of the law decreases with increasing temperature in the FM phase and then exhibits a sharp increase in the HAM phase above $T_{\mathrm{c}}$. This behavior was explained by pinning fields due to the Peierls potential, which depends on sixfold basal anisotropy and a turn angle of the HAM structure in the FM and HAM phases, respectively.

\section{ACKNOWLEDGMENT}

This research was partly supported by the President's research fund of Iwate University.

\footnotetext{
"koba@iwate-u.ac.jp

${ }^{1}$ J. P. Liu, E. Fullerton, O. Gutfleisch, and D. J. Sellmyer, Nanoscale Magnetic Materials and Applications (Springer, New York, 2009).

${ }^{2}$ G. Bertotti, Hysteresis in Magnetism (Academic, San Diego, 1998). ${ }^{3}$ C. P. Steinmetz, Proc. IEEE 72, 197 (1984).

${ }^{4}$ F. J. G. Landgraf, M. Emura, and M. F. de Campos, J. Magn. Magn. Mater. 320, e531 (2008).

${ }^{5}$ S. Kobayashi, S. Takahashi, T. Shishido, Y. Kamada, and

H. Kikuchi, J. Appl. Phys. 107, 023908 (2010).
}

${ }^{6}$ S. Kobayashi, A. Saito, S. Takahashi, Y. Kamada, and H. Kikuchi, Appl. Phys. Lett. 92, 182508 (2008).

${ }^{7}$ S. Kobayashi, N. Kikuchi, S. Takahashi, Y. Kamada, and H. Kikuchi, J. Appl. Phys. 108, 043904 (2010).

${ }^{8}$ S. Kobayashi, Phys. Rev. Lett. 106, 057207 (2011).

${ }^{9}$ E. Loffler and H. Rauch, J. Phys. Chem. Solids 30, 2175 (1969).

${ }^{10}$ A. Del Moral and E. W. Lee, J. Phys. F 4, 280 (1974).

${ }^{11}$ S. B. Palmer, J. Phys. F 5, 2370 (1975). 
${ }^{12}$ T. J. McKenna, S. J. Campbell, D. H. Chaplin, and G. V. H. Wilson, J. Phys. Condens. Matter 3, 1855 (1991).

${ }^{13}$ S. Legvold, in Ferromagnetic Materials, edited by E. P. Eohlfarth (North-Holland, Amsterdam, 1986), p. 183.

${ }^{14}$ C. P. Herring and J. P. Jakubovics, J. Phys. F 3, 157 (1973).

${ }^{15}$ W. D. Corner, F. M. Saad, D. W. Jones, and R. G. Jordan, in Rare Earths and Actinides, edited by W. D. Corner and B. K. Tanner (Inst. Phys. Publishing, Bristol, 1978), p. 222.

${ }^{16}$ R. L. Smith, W. D. Corner, and B. K. Tanner, J. Magn. Magn. Mater. 20, 265 (1980).

${ }^{17}$ W. D. Corner, H. Bareham, R. L. Smith, F. M. Saad, B. K. Tanner, S. Farrant, D. W. Jones, B. J. Beaudry, and K. A. Gschneidner Jr., J. Magn. Magn. Mater. 15-18, 1488 (1980).
${ }^{18}$ T. J. McKenna, S. J. Campbell, D. H. Chaplin, and G. V. H. Wilson, J. Magn. Magn. Mater. 124, 105 (1993).

${ }^{19}$ J. Bindels, J. Bijvoet, and G. W. Rathenau, Physica 26, 163 (1960).

${ }^{20}$ J. Baruchel, G. Aubert, S. B. Palmer, and M. Schlenker, J. Magn. Magn. Mater. 54-57, 1631 (1986).

${ }^{21}$ S. B. Palmer, J. Baruchel, A. Drillat, C. Patterson, and D. Fort, J. Magn. Magn. Mater. 54-57, 1626 (1986).

${ }^{22}$ T. Egami and C. D. Graham Jr., J. Appl. Phys. 42, 1299 (1971).

${ }^{23}$ J. J. Rhyne and A. E. Clark, J. Appl. Phys. 38, 1379 (1967).

${ }^{24}$ W. M. Fairbairn and S. Singh, J. Magn. Magn. Mater. 54-57, 867 (1986).

${ }^{25}$ P. I. Menichuk, A. N. Bogdanov, U. K. Rößler, and K.-H. Müller, J. Magn. Magn. Mater. 248, 142 (2002). 